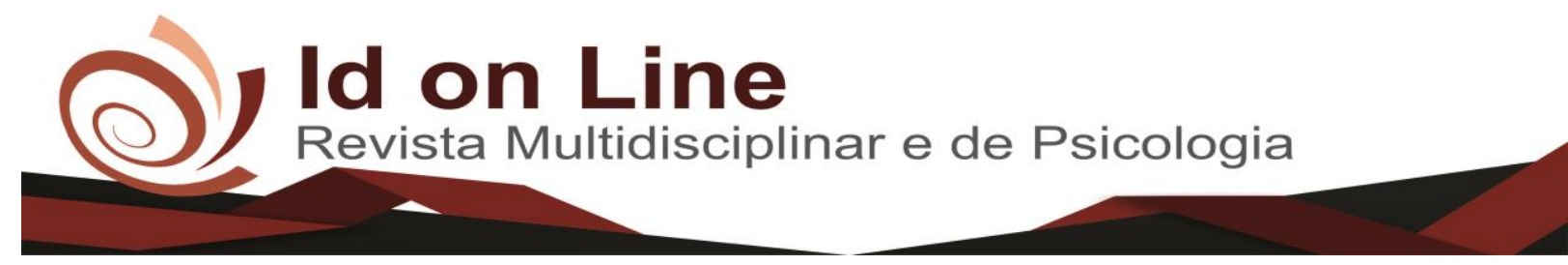

\title{
Qualidade de Vida no Trabalho dos Analistas de Crédito Bancário em Vitória da Conquista/BA
}

\author{
Catilira Gonçalves Santos $^{1}$; Leonardo Costa Sampaio ${ }^{2}$
}

\begin{abstract}
Resumo: A categoria bancária tem sido alvo das mudanças decorrentes da reestruturação dos processos de trabalho e das mudanças nos modelos de gestão que foram aplicadas de forma mais abrangente. Desta forma, o ambiente de trabalho frequentemente apresenta situações geradoras de estresse, em que o indivíduo avalia as exigências do ambiente como uma sobrecarga e podendo afetar o seu bem-estar psicológico e físico. O objetivo geral do estudo é avaliar a qualidade de vida no trabalho dos analistas de crédito bancário e os objetivos específicos são: Traçar o perfil socioeconômico e sociodemográfico da população estudada; Identificar os principais sintomas referentes ao estresse no ambiente de trabalho; avaliar a qualidade de vida dos analistas de crédito; verificar quais atividades os participantes da pesquisa realizam fora do trabalho. A metodologia caracteriza - se como descritiva e exploratória. Essa pesquisa foi realizada com analistas de crédito bancário da cidade de Vitória da Conquista, Bahia. Utilizou-se o questionário validado Medical Outcomes Study 36 - Item Short - Form Health Survey para testar a qualidade de vida, além de uma ficha de perfil do participante e uma entrevista relacionada à sua rotina fora do ambiente de trabalho. Os resultados deste trabalho contribuem de forma positiva e construtiva para o tema da qualidade de vida, pois quantifica as suspeitas e cria perfis no que diz respeito ao estresse e a sua relação com a rotina de trabalho. Conclui-se que a limitação por aspectos físicos e a dor são os elementos que mais agravam a qualidade de vida no trabalho da amostra, o que aponta para a necessidade de propor estratégias de enfrentamento e a melhora no ambiente de trabalho como a diminuição dos fatores estressores.
\end{abstract}

Palavras-Chave: Analistas. Qualidade de vida. Trabalho.

\section{Quality of Life in the Analysts Workers of a Bank Credit in Vitória da Conquista, State of Bahia}

\begin{abstract}
The banking category has been the target of the changes resulting from the restructuring of work processes and the changes in the management models that have been applied more comprehensively. In this way, the work environment often presents situations generating stress, in which the individual evaluates the demands of the environment as an overload and can affect his psychological and physical well-being. The general objective of the study is to evaluate the quality of life in the work of bank credit analysts and the specific objectives are: To trace the socioeconomic and sociodemographic profile of the studied population; Identify the main symptoms related to stress in the work environment; evaluate the quality of life of credit analysts; check what activities the research participants do out of work. The methodology is characterized as descriptive and exploratory. This research was carried out with banking credit analysts from the city of Vitória da Conquista, Bahia. The Medical Outcomes Study 36 - Item Short - Form Health Survey validated questionnaire was used to test the quality of life, as well as a participant's profile card and an interview related to his / her routine outside the work environment. The results of this work contribute in a positive and constructive way to the theme of quality of life, as it quantifies the suspicions and creates profiles regarding stress and its relation with the work routine. It was concluded that physical aspects limitation and pain are the elements that increase the quality of life in the sample work, which points to the need to propose coping strategies and the improvement in the work environment as the reduction of the factors stressors.
\end{abstract}

Key words: Analysts. Quality of life. Work.

\footnotetext{
${ }^{1}$ Graduanda em Fisioterapia pela Faculdade Independente do Nordeste, autor para correspondência. Avenida Deputado Luiz Eduardo Magalhães, 1305, Vitória da Conquista -BA, 45055-030.

Email: catilira011@ @otmail.com;

${ }^{2}$ Professor Especialista do curso de Fisioterapia pela Faculdade Independente do Nordeste. 


\section{Introdução}

A categoria bancária tem sido alvo das mudanças decorrentes da reestruturação dos processos de trabalho e das mudanças nos modelos de gestão que foram implementadas de forma mais abrangente. Essas mudanças transformaram o contexto do setor bancário, onde a intensificação do trabalho, o estimulo à competitividade entre colegas, a ameaça de desemprego, as exigências constantes de qualificação, entre outros, que passaram a fazer parte do cotidiano do trabalhador bancário (RIBEIRO, 2010).

Desta forma, o ambiente de trabalho frequentemente apresenta situações geradoras de estresse, situações estas em que o indivíduo avalia as exigências do ambiente como uma sobrecarga o que pode afetar o seu bem-estar psicológico e físico. As pessoas são de certa forma a base estrutural de qualquer organização e como tal devem ser percebidas, devendo ser reconhecidas e valorizadas de acordo com as necessidades humanas, usando fatores motivacionais e benefícios (LIMONGI-FRANÇA, 2012).

O trabalhador é o suporte do sistema produtivo, e precisa enfrentar estas mudanças apresentando maior capacidade de adaptação e assimilação de tudo que é novo, criatividade e compreensão da realidade, ao mesmo tempo em que constrói sua história e sua cultura com valores adquiridos ao longo da vida. Do outro lado, a própria organização que exige, não oferece, vive atrelada a modelos burocráticos de gestão que buscam cada vez mais produtividade e tem dificuldades de se auto avaliar e mudar, exigindo cada vez mais resultados do trabalhador (FRANÇA; RODRIGUES, 2011).

A crítica ao trabalho que visa essencialmente à máxima produtividade vem ocorrendo há muito tempo, sendo debatida com mais ênfase nos dias atuais. Um esquema de trabalho que busca única e exclusivamente o aumento da produtividade, pode por um curto período ser confundido com um modelo de gestão de sucesso, mas também pode levar a resultados negativos que superam em muito as vantagens que possam ter sido alcançadas (NETTO, 2010).

Em paralelo, verificamos que o conceito de estresse vem sendo amplamente utilizado, não apenas em pesquisas científicas, mas também pelos meios de comunicação. Este uso tão difundido faz com que o conceito seja empregado, muitas vezes, no sentido do senso comum. Na verdade, as reações de estresse são naturais e necessárias para a própria vida, às vezes podem tornar-se prejudiciais ao funcionamento dos sistemas orgânico e psicológico do indivíduo (FRANÇA; RODRIGUES, 2011). 
Dentre os tipos de estresse, está o estresse ocupacional que se caracteriza como um estado desagradável decorrente de aspectos do trabalho, que o indivíduo considera ameaçadores à sua autoestima e ao seu bem-estar. É necessário compreender o estresse ocupacional como um processo composto por inúmeras variáveis, dos fatores ambientais aos psicossociais e pessoais, não atribuindo como fator único nem o ambiente nem o indivíduo (PIAZI, 2012).

Nessa perspectiva, a prevenção representa a principal ação para que tais riscos profissionais sejam evitados ou diminuídos. Percebe-se a importância de medidas de proteção que se inicia com a identificação e avaliação das condições de trabalho, através da análise do corpo bancário das agências em questão, do ponto de vista de sua saúde e qualidade de vida. Sendo que o conhecimento da realidade desses profissionais no exercício de sua função possibilita medidas que possam prevenir os efeitos negativos na saúde, que o sobre-esforço na realização de tarefas pode ocasionar a esses funcionários (NETTO, 2010).

Dessa forma o objetivo geral do estudo é avaliar a qualidade de vida no trabalho dos analistas de crédito bancário e os objetivos específicos são: Traçar o perfil socioeconômico e sociodemográfico da população estudada; Identificar os principais sintomas referentes ao estresse no ambiente de trabalho; avaliar a qualidade de vida dos analistas de crédito; verificar quais atividades os participantes da pesquisa realizam fora do trabalho.

\section{Materiais e Métodos}

Trata-se de um estudo exploratório transversal, logo que é feita em um recorte de tempo e de abordagem quantitativa. É descritivo observacional e de corte transversal, pois observa, descreve e registra o perfil dos sujeitos de pesquisa e a percepção destes a respeito da qualidade de vida no trabalho.

Este trabalho foi feito com analistas de crédito bancário da cidade de Vitória da Conquista, no estado da Bahia. Os analistas de crédito bancário foram escolhidos por passar por situações de pressão tanto por parte da empresa quanto por parte dos clientes, o primeiro cobra a produtividade e o segundo cobra agilidade na liberação de seu crédito. Os sujeitos da pesquisa foram escolhidos randomicamente. De um universo formado por 20 analistas de crédito, apenas 1 (um) não se encaixou no critério de inclusão por exercer a função há menos de 6 (seis) meses. Assim, a amostra se restringiu a 19 indivíduos. 
Utiliza-se o questionário validado Medical Outcomes Study 36 - Item Short - Form Health Survey ( $S F-36$ ), uma entrevista para verificar sua rotina fora do ambiente de trabalho e, para o mapeamento do perfil da população, organizou-se uma ficha de perfil. O SF-36 é um instrumento genérico de avaliação da qualidade de vida, de fácil administração e compreensão. Consiste em um questionário multidimensional formado por 36 (trinta e seis) itens, englobados em 8 (oito) escalas ou domínios, que são: capacidade funcional, aspectos físicos, dor, estado geral da saúde, vitalidade, aspectos sociais, aspectos emocionais e saúde mental. Apresenta um escore final de 0 (zero) á 100 (cem), onde o zero corresponde ao pior estado geral de saúde e o 100 corresponde ao melhor estado de saúde.

A população foi definida da seguinte forma:

a) Exerce a função de analista de crédito bancário há mais de 6 (seis) meses;

b) Nunca tenha sido afastado do trabalho por LER/DORT e/ou outras doenças ocupacionais;

c) Deficientes visuais;

Neste sentido, separar-se-á a amostra da população a partir destes critérios, sendo a e b serão critérios de inclusão enquanto que c será critério de exclusão.

Apenas após a aprovação pelo Comitê de Ética da Instituição sob o número do parecer: 2.960.911, e após todos os participantes assinarem o termo de consentimento livre e esclarecido (TCLE), deu-se início ao trabalho. Os dados coletados pelos questionários receberam tratamento descritivo (média, desvio padrão e prevalências), através do software Statistical Package for the Social Sciences- SPSS 22.0 para o windowns. As tabelas foram plotadas no Excel.

\section{Resultados}

De um universo formado por 20 analistas de crédito, apenas 1 (um) não se encaixou no critério de inclusão por exercer a função há menos de 6 (seis) meses. Assim, a amostra se restringiu a 19 indivíduos. A maioria dos analistas de créditos aqui estudado são do sexo masculino $10(52,6)$, com faixa etária predominantemente entre 31 e 35 anos, $8(42,1 \%)$, de cor parda 9 (47,36\%), de estado civil casado $7(36,84 \%)$ e com ensino médio completo em 11 $(57,89 \%)$ dos casos (Tabela 1). 
Tabela 1. Características sociodemográficas da amostra. Vitoria da Conquista - BA, 2018.

\begin{tabular}{|c|c|c|c|}
\hline Características & $\%$ em resposta & $\mathbf{n}$ & $\%$ \\
\hline Sexo & 100 & & \\
\hline Feminino & & 9 & 47,4 \\
\hline Masculino & & 10 & 52,6 \\
\hline Faixa etária, anos & 100 & & \\
\hline $18-25$ & & 2 & 10,5 \\
\hline $26-30$ & & 5 & 26,3 \\
\hline $31-35$ & & 8 & 42,1 \\
\hline$\geq 36$ & & 4 & 21,1 \\
\hline Cor & 100 & & \\
\hline Branca & & 6 & 31,57 \\
\hline Amarela & & 2 & 10,52 \\
\hline Parda & & 9 & 47,36 \\
\hline Negro & & 2 & 10,52 \\
\hline Estado civil & 100 & & \\
\hline Solteiro & & 5 & 26,31 \\
\hline Casado & & 7 & 36,84 \\
\hline Divorciado & & 4 & 21,05 \\
\hline União Estável & & 3 & 15,78 \\
\hline Escolaridade & 100 & & \\
\hline Fundamental completo & & 1 & 5,26 \\
\hline Ensino médio completo & & 11 & 57,89 \\
\hline Ensino médio incompleto & & 5 & 26,31 \\
\hline Ensino superior incompleto & & 2 & 10,52 \\
\hline
\end{tabular}

${ }^{1}$ Média; ${ }^{2}$ Desvio padrão amostral; Fonte: Dados da pesquisa.

Os participantes da pesquisa responderam à entrevista semi-estruturada objetiva contendo situações dos campos emocionais e físicos. A prevalência das respostas está apresentada na tabela 2 . 
Tabela 2. Características referidas quanto ao stress no ambiente de trabalho. Vitoria da Conquista-BA, 2018.

\begin{tabular}{|c|c|c|c|c|c|}
\hline Características & $\begin{array}{c}\% \\
\text { respostas } \\
\end{array}$ & Nunca & $\begin{array}{c}\text { Algumas } \\
\text { vezes }\end{array}$ & $\begin{array}{c}\begin{array}{c}\text { Frequentemen } \\
\text { te }\end{array} \\
\end{array}$ & $\begin{array}{c}\text { Sempr } \\
\text { e }\end{array}$ \\
\hline Questões físicas & 100 & & & & \\
\hline Fadiga & & $\begin{array}{c}3 \\
(15,78)\end{array}$ & $12(63,15)$ & $3(15,78)$ & $\begin{array}{c}1 \\
(5,26)\end{array}$ \\
\hline Doenças recorrentes & & $\begin{array}{c}4 \\
(21,05)\end{array}$ & $12(63,15)$ & $3(15,78)$ & - \\
\hline Problemas de sono & & $\begin{array}{c}2 \\
(10,52)\end{array}$ & $12(63,15)$ & $5(26,31)$ & - \\
\hline Problemas alimentares & & $\begin{array}{c}3 \\
(15,78)\end{array}$ & $16(84,21)$ & - & - \\
\hline Questões emocionais & 100 & & & & \\
\hline Irritabilidade extrema & & $\begin{array}{c}4 \\
(21,05)\end{array}$ & $10(52,63)$ & $5(26,31)$ & - \\
\hline Falta de Concentração & & $\begin{array}{c}7 \\
(36,84)\end{array}$ & $11(57,89)$ & $1(5,26)$ & - \\
\hline Pessimismo & & $\begin{array}{c}7 \\
(36,84)\end{array}$ & $9(47,36)$ & $3(15,78)$ & - \\
\hline $\begin{array}{l}\text { Dificuldade de } \\
\text { comunicação }\end{array}$ & & $\begin{array}{l}10(52,6 \\
3)\end{array}$ & $7(36,84)$ & $2(10,52)$ & - \\
\hline Redução do potencial criativo & & $\begin{array}{c}10(52,6 \\
3)\end{array}$ & $7(36,84)$ & $2(10,52)$ & - \\
\hline $\begin{array}{l}\text { Redução da } \\
\text { produtividade }\end{array}$ & & $\begin{array}{c}5 \\
(26,31)\end{array}$ & $12(63,15)$ & $2(10,52)$ & - \\
\hline
\end{tabular}

Fonte: Dados da pesquisa.

Os analistas de crédito afirmam que em algumas vezes $12(63,15 \%)$ sentem fadiga, têm problemas com doenças recorrentes e problemas do sono. Em 16 (84,21\%) dos casos afirmam terem problemas alimentares. Nas questões emocionais, os indivíduos disseram em 10 $(52,63 \%)$ dos casos terem passado algumas vezes por problemas de irritabilidade extrema e 11 (57,89\%) falta de concentração. Pessimismo é um sentimento referido como algumas vezes em $9(47,36 \%)$ dos casos. A maioria da amostra $10(52,63 \%)$ afirmou nunca ter tido dificuldade de comunicação relacionado com estresse no ambiente de trabalho, assim como nunca passaram por redução do potencial criativo em $10(52,63 \%)$ dos casos. Entretanto, $12(63,15 \%)$ afirmaram terem passado por situação de redução da produtividade apenas algumas vezes.

A qualidade de vida da amostra foi analisada com o SF-36. Os domínios variam de 0 a 100, onde se considera que quanto maior a pontuação, melhor a qualidade de vida. Os dados estão compilados na tabela 3: 
Tabela 3. Qualidade de vida dos analistas de crédito. Vitória da Conquista - BA, 2018.

\begin{tabular}{|c|c|c|c|c|}
\hline \multicolumn{2}{|c|}{ Domínios } & \multirow{2}{*}{$\frac{\text { Média } \pm \text { Desvio padrão }}{63,68 \pm 21,39}$} & \multirow{2}{*}{$\frac{\text { Mínimo }}{25}$} & \multirow{2}{*}{$\frac{\text { Máximo }}{100}$} \\
\hline$S F-36^{1}$ & Capacidade funcional & & & \\
\hline & Limitação por aspectos físicos & $42,10 \pm 34,41$ & 0 & 100 \\
\hline & Dor & $50,21 \pm 16,62$ & 20 & 100 \\
\hline & Estado geral de saúde & $56,78 \pm 14,65$ & 25 & 82 \\
\hline & Vitalidade & $55 \pm 16,49$ & 30 & 100 \\
\hline & Aspectos sociais & $57,89 \pm 17,78$ & 25 & 100 \\
\hline & Limitação por aspectos emocionais & $57,03 \pm 33,03$ & 0 & 100 \\
\hline & Saúde mental & $64,21 \pm 15,23$ & 40 & 100 \\
\hline
\end{tabular}

136-Item Short Form Health Survey; Fonte: Dados da pesquisa.

A qualidade de vida dos analistas de crédito está melhor nos domínios capacidade funcional e saúde mental, com médias acima de 60 pontos. Os domínios Estado geral da saúde, vitalidade, aspectos sociais e limitações por aspectos sociais apresentam média entre 55 e 60 pontos. Dor é o segundo pior domínio da qualidade de vida, com pouco mais que 50 pontos. E a qualidade de vida se tratando de limitações por aspectos físicos é o pior domínio, com casos em que o indivíduo atingiu 0 no escore geral, cuja média entre todos os participantes ficou em $42,10 \pm 34,41$ pontos.

A tabela 4 reúne os dados sobre quais atividades fora do trabalho os analistas de crédito realizam.

Tabela 4. Atividades realizadas além das atividades de trabalho. Vitoria da Conquista - BA, 2018.

\begin{tabular}{|c|c|c|c|c|c|}
\hline Características & $\begin{array}{c}\% \\
\text { respostas }\end{array}$ & Nunca & $\begin{array}{l}\text { Algumas } \\
\text { vezes }\end{array}$ & $\begin{array}{c}\text { Frequentemen } \\
\text { te }\end{array}$ & $\begin{array}{c}\text { Sempr } \\
\text { e }\end{array}$ \\
\hline Atividades & 100 & & & & \\
\hline Praticar esportes & & $\begin{array}{c}6 \\
(31,57)\end{array}$ & $12(63,15)$ & $1(5,26)$ & - \\
\hline Alimentação saudável & & - & $14(73,68)$ & $5(26,31)$ & - \\
\hline Ter tempo para lazer & & $\begin{array}{c}2 \\
(10,52)\end{array}$ & $7(36,84)$ & $6(31,57)$ & $\begin{array}{c}4 \\
(21,05)\end{array}$ \\
\hline $\begin{array}{l}\text { Realizar atividades } \\
\text { relaxantes }\end{array}$ & & $\begin{array}{c}2 \\
(10,52)\end{array}$ & $7(36,84)$ & $6(31,57)$ & $\begin{array}{c}4 \\
(21,05)\end{array}$ \\
\hline $\begin{array}{l}\text { Controlar abusos (consumo d } \\
\text { álcool e tabagismo) }\end{array}$ & & - & $10(52,63)$ & $5(26,31)$ & $\begin{array}{c}4 \\
(21,05) \\
(-2\end{array}$ \\
\hline
\end{tabular}


A prática de esportes é referida como realizada em algumas vezes, por 12 (63,15\%), mas $6(31,57 \%)$ dizem não realizar atividade física nunca. A maioria cuida da alimentação 14 $(73,68 \%)$ em algumas vezes dos casos. Ter tempo para lazer e realizar atividades relaxantes é feita em algumas vezes em sua maioria, com 7 (36,84\%) da amostra e se dedicar ao controle de abusos (álcool e tabagismo), em algumas vezes em sua maioria, com 10 (52,63\%) dos entrevistados.

\section{Discussão}

As organizações do século XXI estão inclinadas a buscar uma maior aproximação de seus empregados com a empresa, novos meios para melhorar a vida de seus trabalhadores e fazer com que eles se sintam parte integrante da mesma. Essa aproximação se faz cada vez mais necessária nas organizações, em virtude das diversas expectativas que os indivíduos têm ao se inserirem no mercado de trabalho (MAXIMIANO, 2010)

Com foco neste pensamento é que empresas socialmente responsáveis procuram aliar razão e sentimento a favor do próprio crescimento organizacional. Um novo ambiente de trabalho que proporcione melhor convivência, amenizando conflitos interpessoais, são reivindicações da classe economicamente ativa para redução das situações de estresse que podem torná-la menos produtiva e susceptível às doenças ocupacionais (ROBBINS, 2010).

Os dados dessa pesquisa apontam para o estresse, irritabilidade extrema e problemas do sono enquanto fatores agravantes da qualidade do sono. Mais da metade da amostra apresentou esses motivos enquanto principais e mais recorrentes na jornada de trabalho.

Para que estas transformações aconteçam no ambiente, é preciso o controle das ações que possam estar ligadas a esses conflitos, implantando programas de combate ao estresse e incentivo ao lazer a fim de proporcionar essa nova perspectiva de vida. Partindo desse princípio, qualidade no ambiente de trabalho não é mais uma vantagem concedida a uma ou mais pessoas, é sim uma obrigação para essas empresas que estão preocupadas em implementar programas internos de saúde ocupacional que proporcionem mais felicidade e satisfação para os trabalhadores, mesmo por que, traz como retorno o comprometimento e envolvimento dos empregados com os objetivos da empresa (VIEIRA, 2010).

Em analogia a sequência de atitudes burocráticas, o teor expressivo do trabalho é normalmente posto de lado em detrimento de outras prioridades, associadas ao controle e 
resultados, tornando inviável o incremento de processos produtivos com forte conteúdo de importância e motivação para os trabalhadores. O sofrimento psíquico ao descontentamento no trabalho, aliado ao processo produtivo e a experiência de vida dos trabalhadores nas relações de trabalho, pode gerar sentimentos de depressão e redução da autoestima (LIMONGIFRANÇA, 2012).

O trabalho ocupa a maior parte do tempo do ser humano. É o ponto central da vida do homem. Cada indivíduo passa a maior parte de sua vida em seu local de trabalho, dedicando sua força, energia e esforços para as organizações. Ou seja, disponibilizando a maior parte do seu tempo ao trabalho do que propriamente com suas famílias e amigos (NETTO, 2010).

Atualmente as organizações vêem a necessidade de investir em programas de qualidade de vida no trabalho, visando uma melhora no bem-estar dos funcionários, de sua capacidade produtiva e, consequentemente, de seus resultados. Há uma unanimidade na certeza que o fator humano é o principal elemento diferenciador, e o agente responsável pelo sucesso de todo e qualquer negócio. As empresas para alcançarem a satisfação de seus clientes precisam ter seus funcionários envolvidos com o objetivo da organização e satisfeitos (FERNANDES, 2013).

Nesse sentido, uma possibilidade de melhorar a qualidade de vida dos trabalhadores seriam investimentos no incentivo às práticas esportivas e na reeducação alimentar para uma alimentação saudável, logo que, em nossos dados, nenhum sujeito tem uma dessas práticas enquanto uma prática sempre frequente.

É importante refletir sobre a Qualidade de Vida no Trabalho (QVT) lembrando sempre a importância deste tema como questão de competitividade organizacional. Logo, é relevante o desenvolvimento de elementos que beneficiem o atendimento e o desenvolvimento do ser humano como um ser complexo. Reconhecendo suas mais variadas necessidades. Vale ressaltar que o ser humano tem uma diversidade de sentimentos e ambições. Cria expectativas e geralmente vive busca de crescimento dentro daquilo que desenvolve e realiza (FERNANDES, 2013).

As criações de novas ferramentas de gestão proporcionam o surgimento de novas propostas e aperfeiçoamento nas antigas propostas, mas deixam a desejar naquelas que visam proporcionar melhor condição de trabalho e satisfação na sua execução.

Os benefícios da qualidade de vida gerados para a empresa podem ser listados como a melhoria na sua produtividade diária; empregados motivados e mais alertas; baixas taxas de 
enfermidades; redução na fadiga, melhoria do clima organizacional e a imagem corporativa; menos absenteísmo; aumento dos níveis de satisfação e saúde do colaborador e consumidor; beneficia o processo de formação e desenvolvimento humano agregando competência aos mesmos (NETTO, 2010).

A QVT pode ser estruturada com base em oito dimensões: compensação justa e adequada, condições de trabalho, oportunidade de uso e desenvolvimento de capacidades, oportunidade de crescimento e segurança, interação social na organização, constitucionalismo, trabalho e vida e relevância social da vida no trabalho. Baseando-se na proposta apresentada por Walton, a QVT pode ser tomada como uma expressão para caracterizar determinados valores ambientais e humanos que foram supridos em favor do avanço tecnológico, da produtividade industrial e do crescimento econômico (RODRIGUES, 2014).

\section{Conclusão}

Conclui-se que a limitação por aspectos físicos e a dor são os elementos que mais agravam a qualidade de vida no trabalho da amostra, o que aponta para a necessidade de propor estratégias de enfrentamento e a melhora no ambiente de trabalho como a diminuição dos fatores estressores.

Além de enriquecer os estudos sobre qualidade de vida no trabalho, essa pesquisa tem valor reflexivo tanto para os profissionais de fisioterapia quanto para a área dos analistas de crédito bancário e estudiosos da qualidade de vida no trabalho de modo geral pois contribui com a área e reflete sobre as condições de trabalho desta categoria. Seria de grande importância pesquisas com maior amostra e com o objetivo de verificar qual tipo de prática fisioterapêutica seria mais conveniente para tratar o estresse ocupacional.

\section{Referências}

CHIAVENATO, Idalberto. Gestão de Pessoas; O Novo Papel dos Recursos Humanos na Organização. RJ: Elsevier, 2014.

FEBRABAN. Relatório Anual 2009. Federação Brasileira de Bancos, 2009.

FEBRABAN. Lista de Bancos: Classificação. Disponível em: <http://www.buscabanco.org.br/ AgenciasBancos.asp>. Acesso em: 27 MAI. 2018. 
FERREIRA, A. B. de H. Dicionário Aurélio básico da língua portuguesa. Botafogo,RJ: Nova Fronteira S.A., 2012.

FERNANDES, Eda Conte. Qualidade de Vida no Trabalho. Salvador: Casa da Qualidade, 2013.

FRANÇA, A. C. L.; RODRIGUES, A. L. Stress e trabalho: uma abordagem psicossomática. 4. ed. São Paulo: Atlas, 2011.

GIL, Antonio C. Métodos e Técnicas de Pesquisa Social. 6. ed. São Paulo: Atlas S.A., 2012

LIMONGI-FRANÇA, Ana Cristina. Qualidade de vida no trabalho - QVT: Conceitos e práticas nas empresas da sociedade pós-industrial. 2. ed. - 3. reimpr. - São Paulo: Atlas, 2012.

MATSUDO S, et al. Nível de atividade física na população do Estado de São Paulo: uma análise de acordo com o gênero, idade, nível socioeconômico, distribuição geográfica e de conhecimento. Rev Bras Ciênc Mov. 2012 Out;10(4):41- 50.

MAXIMIANO, A. C. A. Teoria geral da administração. São Paulo: Atlas S.A., 2010.

NETTO, Talita Gava. Análise da Qualidade de Vida no trabalho dos funcionários do setor logístico em uma empresa de transportes no município de Criciúma/SC. 2010. 72 folhas. Monografia do Curso de Administração com linha específica em Comércio Exterior, da Universidade do Extremo Sul Catarinense, UNESC, Criciúma.

PIAZI, M. D. Estresse no trabalho e lesões por esforços repetitivos (LER) em servidores públicos de uma Universidade no Estado do Rio de Janeiro: Estudo Pró-Saúde. 71f. 2012. Dissertação (Mestrado em Saúde Coletiva) - Universidade do Estado do Rio de Janeiro, Rio de Janeiro, 2012.

ORGANIZAÇÃO MUNDIAL DA SAÚDE. Qualidade de vida. 2015.

RIBEIRO, A. de L. Gestão de pessoas. São Paulo: Saraiva, 2010.

ROBBINS, Stephen P. Comportamento Organizacional. 11. Ed. São Paulo: Afiliada, 2010.

RODRIGUES, Marcus Vinícius Carvalho. Qualidade de Vida no Trabalho. 5. ed. Petrópolis, RJ: Vozes, 2014.

VIEIRA, Adriane. A qualidade de vida no trabalho e o controle da qualidade total. Florianópolis: Insular, 2010.

Como citar este artigo (Formato ABNT):

SANTOS, Catilira Gonçalves; SAMPAIO, Leonardo Costa. Qualidade de Vida no Trabalho dos Analistas de Crédito Bancário em Vitória da Conquista/BA. Id on Line Rev.Mult. Psic., 2018, vol.12, n.42, Supl. 1, p. 600-610. ISSN: 1981-1179. 